\title{
Design parameters analysis and verification of angular vibration sensor based on magnetohydrodynamics
}

\author{
Kundong Wang ${ }^{1, *}$, Youwei $\mathrm{Ma}^{1}$, Jianming $\mathrm{Wu}^{2}$, Qinghua $\mathrm{Zhu}^{2}$, Yue $\mathrm{Gu}^{2}$, and Hongli $\mathrm{Qi}^{3}$ \\ ${ }^{1}$ Department of Instrument Engineering, Shanghai Jiao Tong University, Shanghai 200240, PR China \\ ${ }^{2}$ Shanghai Academy of Spaceflight Technology, China Aerospace Science and Technology (CASC), Shanghai 200240, PR China \\ ${ }^{3}$ No.704 Research Institute, CSIC, Shanghai 200031, PR China
}

Received: 17 October 2020 / Accepted: 18 December 2020

\begin{abstract}
The angular vibration is concerned in many fields such as satellite platform, manufacturing equipment for micro-electromechanical systems. However, the angular vibration with a frequency more than $15 \mathrm{~Hz}$ is difficult to be measured by traditional gyroscopes. The angular vibration sensor based on Magnetohydrodynamics can meet the requirements of both wide bandwidth and higher precision. In order to optimize the structure, a response of conducting fluid in the static magnetic field to the angular vibration is modeled in this paper. Based on this model, the sensitivity of the design parameters of magnetic field intensity, conducting fluids' height and width are analyzed to get an optimized parameter for higher precision and bandwidth. A prototype was developed to verify the analysis and optimization. The experiment results showed that the model is accurate with $6.7 \%$ error in lower-cut-off frequency and $1.4 \%$ error in scale factor. It can meet the design requirement of $6-1000 \mathrm{~Hz}$.
\end{abstract}

Keywords: Magnetohydrodynamics / angular vibration sensor / transfer function / parameter analysis / optimization

\section{Introduction}

Posture direction of the payload in a satellite platform has two basic requirements. The first is a very higher precision with an ultra-low noise, and the second is the wide bandwidth to a frequency of $2000 \mathrm{~Hz}$. The traditional gyroscope cannot meet these requirements any longer. At present, measurements of angular vibration with wide bandwidth rely on angular vibration sensors.

There have been several sensors widely used on satellite platforms to measure the micro-radian angular vibration with high precision and wide bandwidth. BEI 8301 Series of angular displacement sensor can measure angular displacement directly based on variable capacity, which was applied widely to land satellite mapping camera, GEOS (Geosynchronous Earth Orbit Satellite), GPS (Global Positioning System), etc. [1] Another type of sensor based on Magnetohydrodynamics (MHD) outputs the angular rate. The "magnetohydrodynamics" is first proposed by Hannes Alfvén and has been studied by many researchers [2-6]. Angular displacement is derived from the angular rate. It has also been employed in many missions such as

\footnotetext{
* Corresponding author: kdwang@sjtu.edu.cn
}

American reflex tests of relay satellite, the most advanced meteorological satellite Goes- $\mathrm{N}$, the Japanese land satellite ALOS, etc. Magnetohydrodynamics is widely used in sensors, such as angular molecular-electronic Sensor [7], diagonal MHD accelerator [8], etc. The major advantages of the sensor based on MHD principle include simple structure, small size, light quality, low-power, insensitive to crossed-axis and linear acceleration, without moving parts, high reliability, impact resistance, well-adapted with temperature, and being able to realize broadband between 1 and $1000 \mathrm{~Hz}$ and high-precision measurements below $1 \mu \mathrm{rad}$ [9-13]. He researched on micro-posture control algorithm of MHD angular velocity sensor combined with mechanical gyroscope end with simulation [14]. Huo studied the principle and designed the magnetic field of prototype $[15,16]$. Xu proposed a parallel magnetic field scheme to design and realize a prototype, and finished the corresponding testing [17], then improved its performance with new methods and structures in follow-up researches [18-21].

However, all these reports did not reveal the precise transfer function from the angular vibration to the voltage output. This transfer function is regarded as a coupled equation of electromagnetic field, conductive flow field and electrical network. The precise mathematic model should 
be built to design and use the sensor well. This paper firstly abstracts the equivalent flow model. And then, the transfer function was derived based on the basic equations of fluid dynamics, Hartmann effect, Ohm's law, and circuit equations. Next, the parameter sensitivity analysis was performed to optimize the design. Finally, a prototype was manufactured to verify the model and the design.

\section{Mathematic models}

The basic principle of MHD microradian angular vibration sensor is shown in Figure 1a. The vacant space of the annular barrel between the extern tube and the inner tube is filled with the conducting liquid (namely mercury ring), and the permanent magnetic circuit is designed to form outgoing radial magnetic field $B$ in the mercury ring. When the sensor has a micro angular vibration $\omega_{i}$ around $Z$ axis, the conducting liquid will hold its position because of its great inertia and low friction between the tube surface and the liquid. In that case, the mercury ring incises magnetic flux to produce the induced electromotive force $V_{0}$ between the upper electrode and the lower electrode.

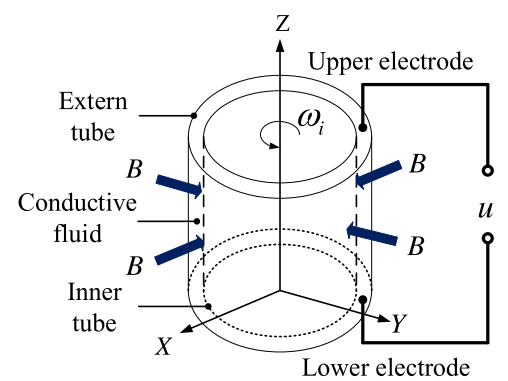

(a)

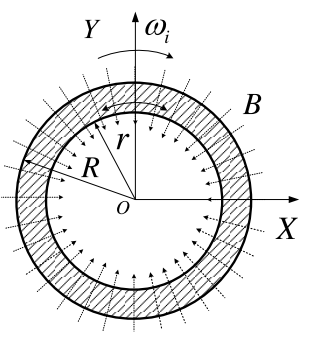

(b)
It is assumed that the conductive fluid's width is one order of magnitude smaller than its length, then the 3D structure of the sensor can be simplified as a $2 \mathrm{D}$ equivalent flow model as shown in Figure 1b. The mercury ring's Inner radius and extern radius are $r$ and $R$, respectively.

Furtherly, the 2D equivalent flow can be expanded along the circumference in order to deduce conveniently. In that case, a plate laminar flow is used to demonstrate the conductive fluid's flow between the extern tube and inner tube as shown in Figure 2. The magnetic field, flow velocity, and electric field are orthogonal to each other governed by the right-hand rule. A micro flow unit is selected to analyze its dynamic model. Initial force $F$, viscous force $F_{u}$ and electromagnetic force $F_{e m}$ are applied on the unit.

According to the Navier-Stokes equation, the force per unit volume can be got as follow.

$$
F=\rho \frac{d v}{d t}=F_{u}+F_{e m}
$$

where, $\rho$ is the density of the conductive liquid, $v$ is the relative velocity of the liquid to the tube. It is assumed that the flow between the two tubes in the perpendicular magnetic field is Hartmann flow. Then, the following equation can be achieved.

$$
\frac{F_{e m}}{F_{u}}=M^{2}
$$

where $M$ is the Hartmann constant. Then equation (1) can be formulated.

$$
\rho \frac{d v}{d t}=\left(1+\frac{1}{M^{2}}\right) F_{e m}
$$

$F_{\text {em }}$ can be calculated by the following.

$$
F_{e m}=-B J_{z}
$$

(a) schematic diagram (b) equivalent flow model to $2 \mathrm{D}$.

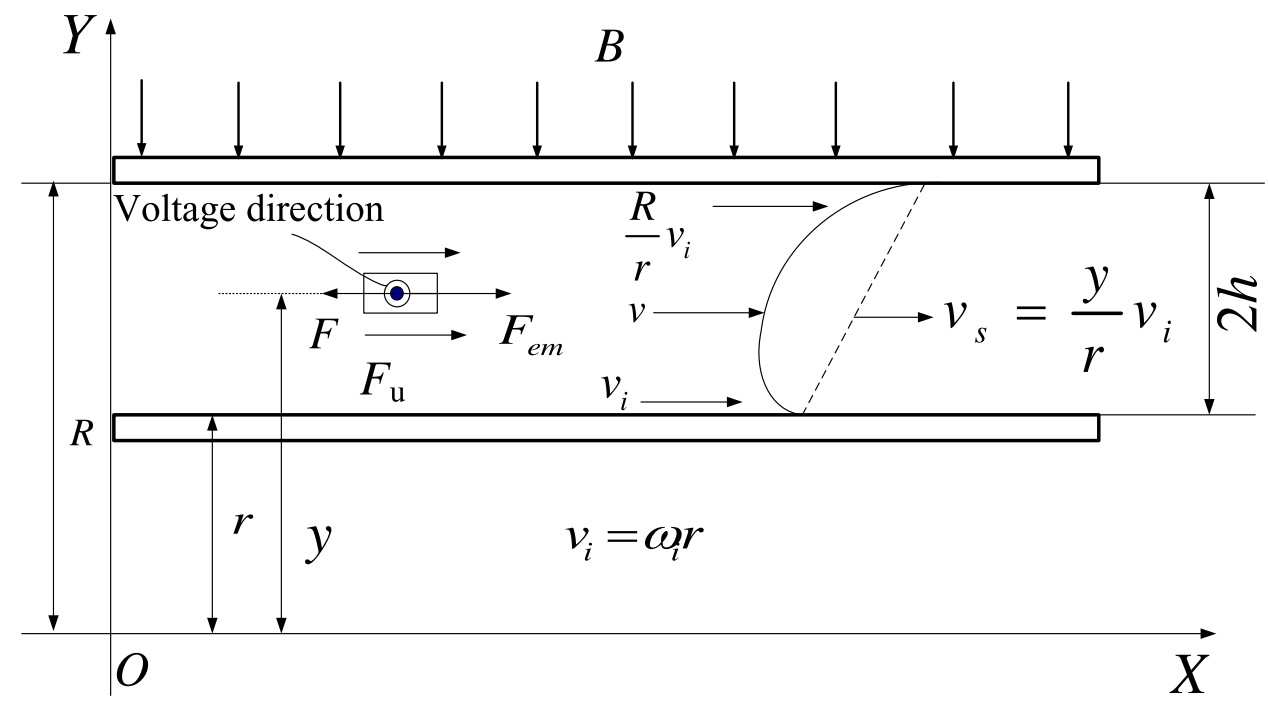

Fig. 2. Equivalent plate laminar flow. 
$J_{z}$ is the current density along $z$ axis. According to the Ohm's law of the whole circuit, $J_{z}$ can be calculated.

$$
J_{z}=\sigma\left(E_{z}-B \times V\right)
$$

where $\sigma$ is the conductivity, $E_{z}$ is the vortex electric field from the varying magnetic field induced by the varying induced current, and $B \times V$ is the induced electric field in the conductive fluid from the cutting magnetic wire. In general, $E_{z}$ is smaller than $B \times V$ because the induced eddy magnetic field is lower three order of magnitudes than the permanent field $B$. The profile of the flow velocity $V$ relative to the magnetic field can be formulated as

$$
V=v_{s}-v=\frac{y}{r} v_{i}-v
$$

where, when $y=r, v=v_{i}$ and when $y=R, v=v_{i} \times R / r$. From equations (4)-(6), we can get

$$
F_{e m}=B^{2} \sigma\left(\frac{y}{r} v_{i}-v\right)
$$

Substituting equation (7) into equation (3), the dynamic equation can be formulated.

$$
\rho \frac{d v}{d t}=\left(1+\frac{1}{M^{2}}\right) B^{2} \sigma\left(\frac{y}{r} v_{i}-v\right)
$$

where $v_{i}=r \omega_{i}$.

After the Laplace Transformation, we get

$$
\rho s v(s)=\left(1+\frac{1}{M^{2}}\right) B^{2} \sigma\left(\frac{y}{r} v_{i}(s)-v(s)\right)
$$

So, we can get $v$

$$
v(s)=\frac{y}{r} v_{i}(s) \frac{1}{B^{2} \sigma\left(1+\frac{1}{M^{2}}\right)}+1
$$

Then the output voltage $U_{z}$ can be formulated

$$
\begin{aligned}
U_{z}(s) & =B l V(s)=B l\left(\frac{y}{r} v_{i}(s)-v(s)\right) \\
& =B l \frac{y}{r} v_{i}(s) \frac{s}{s+\frac{B^{2} \sigma}{\rho}\left(1+\frac{1}{M^{2}}\right)}
\end{aligned}
$$

Because $v_{i}(s)=r \omega_{i}(s)$ and $\mathrm{M}=B \mathrm{~h} \sqrt{\frac{\sigma}{\rho v}}$, the relation between $U_{z}(s)$ and $\omega_{i}(s)$ can be formulated. Here, $v$ is the dynamic viscosity coefficient of the liquid, $h$ is half of the width of hydrogarum loop as shown in Figure 2 [22].

$$
\frac{U_{z}(s)}{\omega_{i}(s)}=B l \frac{s}{s+\frac{v}{\mathrm{~h}^{2}}\left(1+M^{2}\right)} y
$$

where $y$ changes from $r$ to $R$, it can take the mean square root of $R$ and $r$. Then, the transfer function can also be

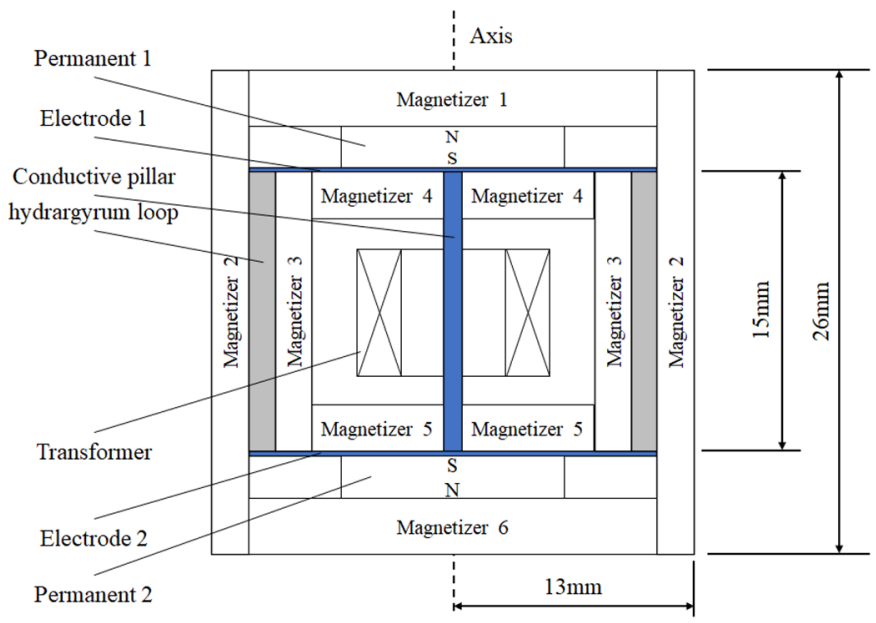

Fig. 3. Mechanism diagram.

formulated as

$$
G(s)=\frac{U_{z}(s)}{\omega_{i}(s)}=B \operatorname{lr}_{R M S} \frac{s}{s+\frac{\nu}{\mathrm{h}^{2}}\left(1+M^{2}\right)}
$$

where $r_{R M S}=\sqrt{\frac{\int_{r}^{R} y^{2} d y}{R-r}}=\sqrt{\frac{\left(R^{2}+R r+r^{2}\right)}{3}}$

\section{Design and analysis}

According to the schematic diagram as shown in Figure 1a, the mechanism is designed as shown in Figure 3. This is a $2 \mathrm{D}$ plane drawing, and the $3 \mathrm{D}$ model can be got by rotating around the axis. Magnetic flux from permanent 1 passes through magnetizer 1, magnetizer 2, hydrargyrum, magnetizer 3, magnetizer 4 , in turn, and finally reaches to permanent 1 . Another magnetic flux from permanent 2 with opposite magnetic field direction configuration also passes through the hydrargyrum in the same direction. The designed total height is $26 \mathrm{~mm}$ and the height of hydrargyrum is $15 \mathrm{~mm}$. The radius is $13 \mathrm{~mm}$, and the inner radius of the hydrargyrum loop is $9.6 \mathrm{~mm}$ and the thickness of the hydrargyrum loop is $1.4 \mathrm{~mm}$. The design can get the radial magnetic field shown in Figure 1b.

Electrodes are installed between the top and the bottom of the hydrargyrum loop and connected through the conductive pillar along the axis as shown in Figure 3. The circuit of hydrargyrum loop, electrodes and conductive pillar can be seen as a primary coil of the transformer. Another coil is installed coaxially with the conductive pillar as the secondary coil of the transformer. This transformer is used to isolate and amplify the signal of the hydrargyrum loop.

The design prototype's parameters are shown in Table 1.

Here, $r_{R M S}=10.31 \mathrm{~mm}$ and $M=15.65$. From Table 1, the transfer function can be recalculated by equation (13) 
Table 1. The designed prototype's parameters.

\begin{tabular}{llll}
\hline Parameter & Symbol & Value & Unit \\
\hline Magnetic flux density & $B$ & 0.7 & $\mathrm{~T}$ \\
Height of hydrogarum loop & $l$ & 15 & $\mathrm{~mm}$ \\
Inner radius of hydrogarum loop & $r$ & 9.6 & $\mathrm{~mm}$ \\
Outer radius of hydrogarum loop & $R$ & 11 & $\mathrm{~mm}$ \\
Width of hydrogarum loop & $\mathrm{h}$ & 0.7 & $\mathrm{~mm}$ \\
Dynamic viscosity coefficient $@ 20{ }^{\circ} \mathrm{C}$ & $v$ & $7.5 \times 10^{-8}$ & $\mathrm{~m}^{2} / \mathrm{s}$ \\
Hydrogarum density & $\rho$ & $13.6 \times 10^{3}$ & $\mathrm{~kg} / \mathrm{m}^{3}$ \\
Conductivity@20 ${ }^{\circ} \mathrm{C}$ & $\sigma$ & $1.04 \times 10^{6}$ & $\mathrm{~s} / \mathrm{m}$ \\
\hline
\end{tabular}

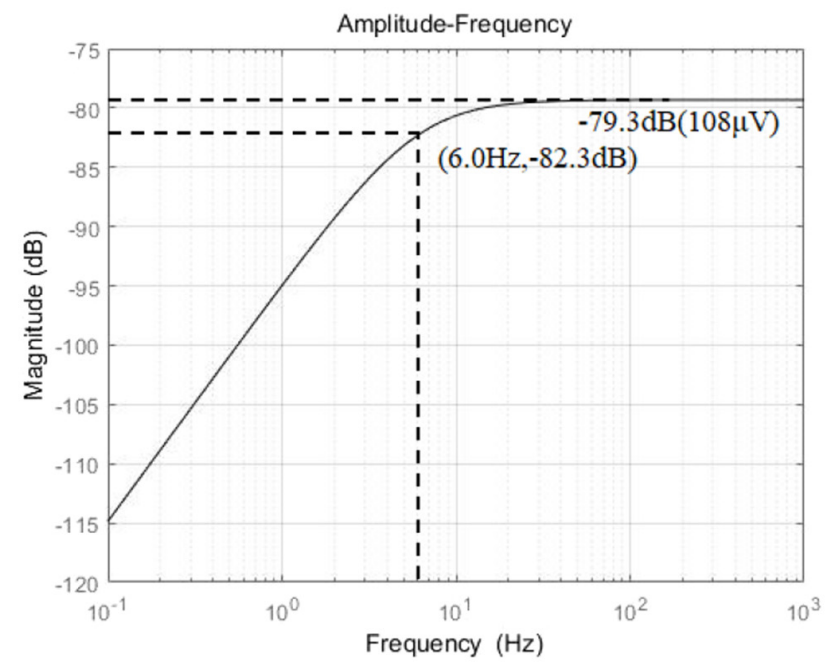

(a)

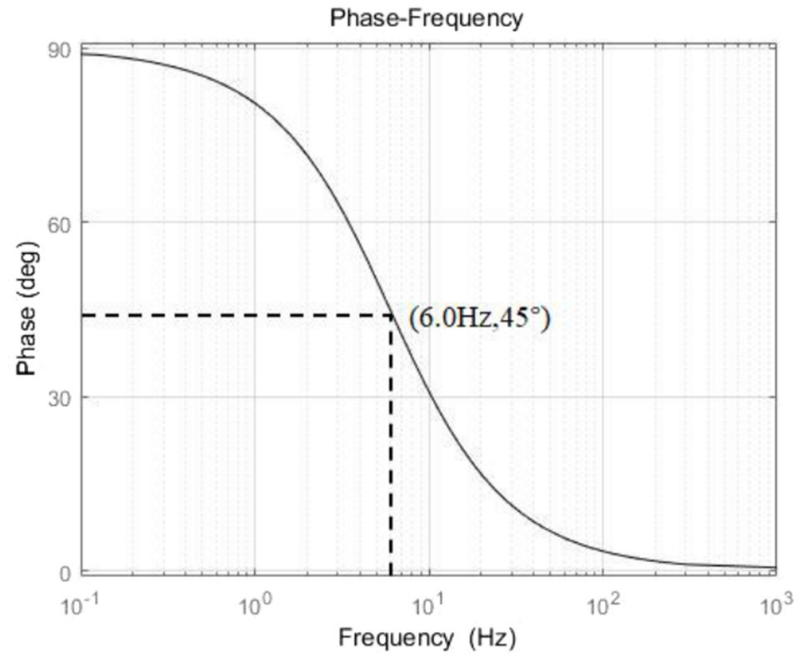

(b)

Fig. 4. Frequency characteristics of MHD angular vibration sensor. (a) Amplitude-frequency responses. (b) Phase-frequency responses.

as the following.

$$
G(s)=\frac{1.082 \times 10^{-4} s}{s+37.624}
$$

According to equation (14), the amplitude-frequency characteristic and the phase-frequency characteristic are shown in Figure 4a and b, respectively. It can be found that the sensor reveals a high pass feature. The corner frequency is about $6.0 \mathrm{~Hz}$ with $-3 \mathrm{~dB}$ attenuation. The output signal's amplitude is about $108 \mu \mathrm{V} / \mathrm{rad} / \mathrm{s}$. Figure $4 \mathrm{~b}$ shows that the phase changes from $90^{\circ}$ to 0 . The phase invert center is $45^{\circ}$ at $6.0 \mathrm{~Hz}$.

Several adjustable parameters related to the design are selected to explore the amplitude-frequency characteristic's sensitivity. These parameters include $B, l, r$, and $R$. When one parameter is adjusted to be an alternation of $\pm 5 \%$ or $\pm 10 \%$, the other parameters are not changed, taking values according to Table 1. Each parameter's influences to the frequency amplitude are shown in Figure 5.
It can be found that within $\pm 10 \%$, the magnetic flux density $B$ and the height of the hydrogarum loop $l$, the inner radius of hydrogarum loop $r$ and the outer radius of the hydrogarum loop $R$ are all positively correlated with the amplitude. However, their influence on the amplitude is different. $B$ and $l$ have the strongest influence, $r$ is less, and $R$ is the least. In addition, $B$ significantly influences the corner frequency. When B increase $10 \%$, the corner frequency increase $20.5 \%$. But $l, r$ and $R$ have no relevance to the corner frequency. The detailed values are listed in Table 2.

\section{Experiments}

The measuring circuit of the sensor is shown in Figure 6 . The signal from the hydrargyrum loop is isolated and amplified through the transformer, then amplified again by an instrumentation amplifier, finally filtered by an active second-order low-pass filter with the cut-off frequency of $1200 \mathrm{~Hz}$. 


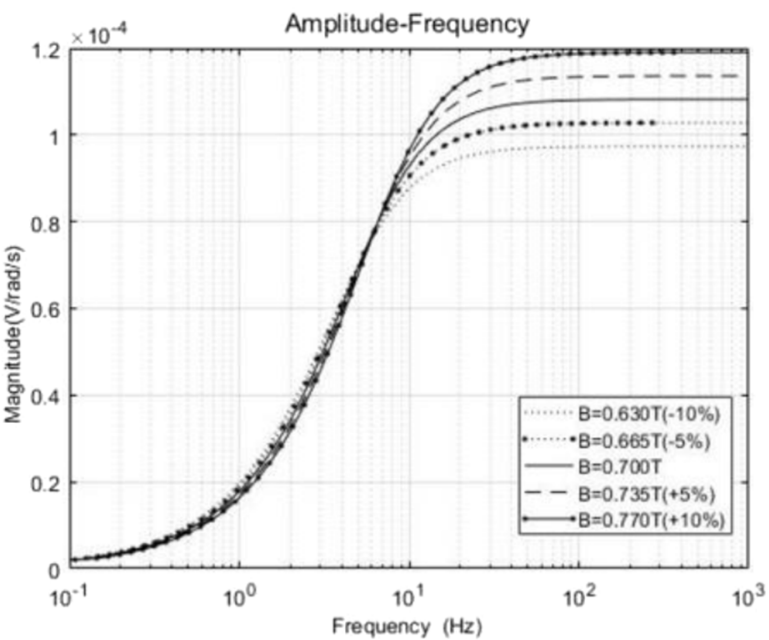

(a)

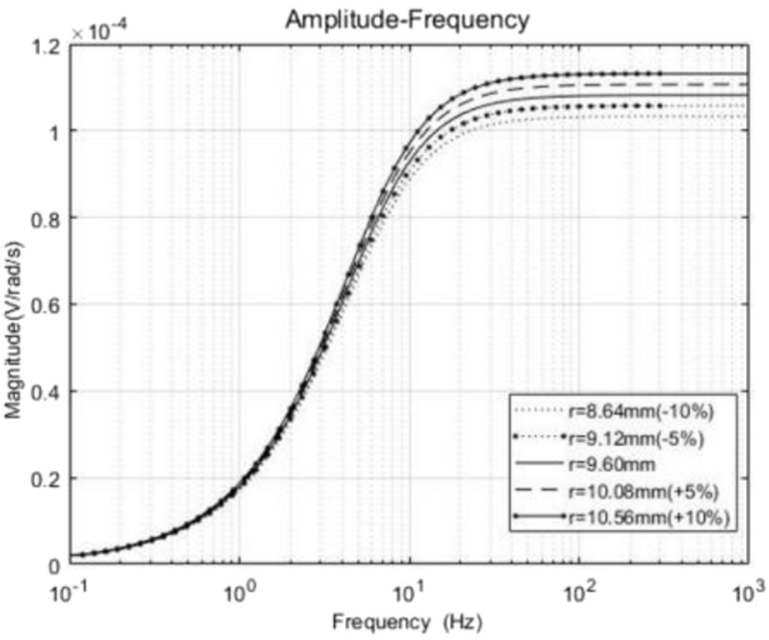

(c)

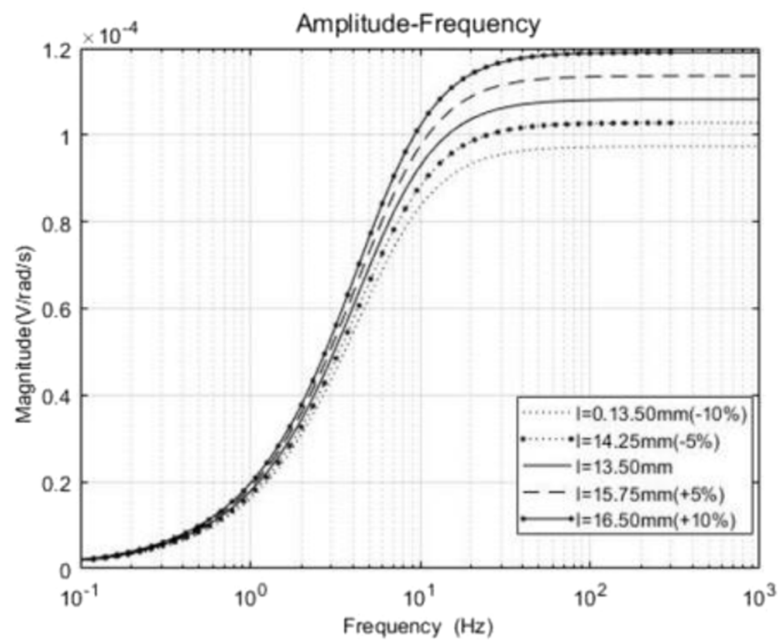

(b)

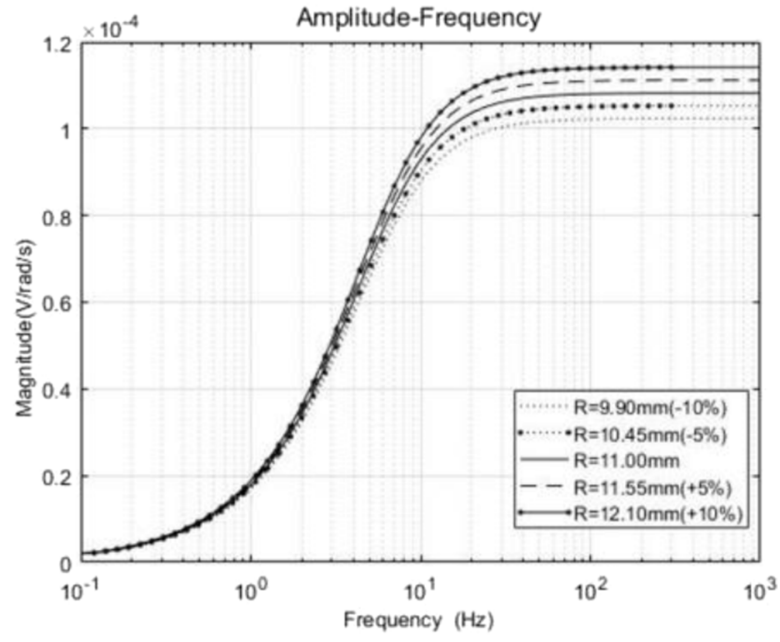

(d)

Fig. 5. The amplitude-frequency characteristic's sensitivity analysis to micro adjustment of the designed parameters. (a) $B$ adjustment; (b) $l$ adjustment; (c) $r$ adjustment; (d) $R$ adjustment.

Table 2. Amplitude and corner frequency changing to parameter alteration.

\begin{tabular}{|c|c|c|c|c|c|c|}
\hline Characteristics & Parameter & $-10 \%$ & $-5 \%$ & 0 & $5 \%$ & $10 \%$ \\
\hline \multirow{4}{*}{ Amplitude $\left(\times 10^{-5} \mathrm{~V} / \mathrm{rad} / \mathrm{s}\right)$} & $B$ & 9.741 & 10.282 & 10.823 & 11.364 & 11.906 \\
\hline & $l$ & 9.741 & 10.282 & 10.823 & 11.364 & 11.906 \\
\hline & $r$ & 10.336 & 10.578 & 10.823 & 11.071 & 11.320 \\
\hline & $R$ & 10.238 & 10.529 & 10.823 & 11.119 & 11.418 \\
\hline \multirow{4}{*}{ Corner frequency $(\mathrm{Hz})$} & $B$ & 4.85 & 5.41 & 6.00 & 6.59 & 7.23 \\
\hline & $l$ & 6.00 & 6.00 & 6.00 & 6.00 & 6.00 \\
\hline & $r$ & 6.00 & 6.00 & 6.00 & 6.00 & 6.00 \\
\hline & $R$ & 6.00 & 6.00 & 6.00 & 6.00 & 6.00 \\
\hline
\end{tabular}




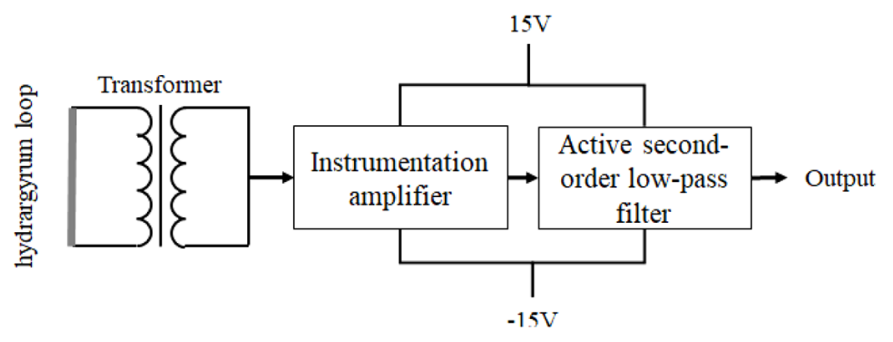

Fig. 6. Measuring circuit.

The scale factor of the prototype is designed to be about $40 \mathrm{~V} / \mathrm{rad} / \mathrm{s}$. As shown in Figure 4, the maximum output of the hydrargyrum loop is $108 \mu \mathrm{V} / \mathrm{rad} / \mathrm{s}$, so the total magnification of the measuring circuit is designed as 370000 . Then the maximum scale factor can be calculated by $108 \mu \times 370000=39.96 \mathrm{~V} / \mathrm{rad} / \mathrm{s}$, close to the target. The prototype of the MHD angular vibration sensor has been made according to the above parameters and the structural design as shown in Figure $7 \mathrm{a}$.

The prototype has been experimented under the frequency of $1-1000 \mathrm{~Hz}$ to test the performance of frequency characteristics as shown in Figure 7b. Since the angular vibration table used in the experiment can only measure under $1000 \mathrm{~Hz}$, the experiment can be only implemented up to $1000 \mathrm{~Hz}$.

The angular vibration used in the experiment is a sine wave with $0.174 \mathrm{rad} / \mathrm{s}\left(10^{\circ} / \mathrm{s}\right) \mathrm{Vpp}$. The original voltage signal of the sensor of every frequency point is sine fitted by

$$
\begin{aligned}
u= & C_{u}\left(\cos \left(2 \pi f \cdot \mathrm{t}+\phi_{u}\right)\right)+D_{u}=A_{u} \cos (2 \pi f \cdot \mathrm{t}) \\
& +B_{u} \sin (2 \pi f \cdot \mathrm{t})+D_{u}
\end{aligned}
$$

where $u$ denotes the original voltage signal of the sensor, $f$ is frequency, $t$ is time, $A_{u}, B_{u}, C_{u}, D_{u}$ are corresponding coefficients, then the output amplitude of the sensor $C_{u}$ and the initial phase $\varphi_{u}$ are

$$
C_{u}=\sqrt{A_{u}^{2}+B_{u}^{2}}, \varphi_{u}=\left\{\begin{array}{l}
\tan ^{-1}\left(\frac{-B_{u}}{A_{u}}\right), A_{u} \geq 0 \\
\tan ^{-1}\left(\frac{-B_{u}}{A_{u}}\right)+\pi, A_{u} \leq 0
\end{array}\right.
$$

The scale factor $S_{\hat{\dot{\theta}}}$ and phase delay $\Delta \phi_{\dot{\theta}}$ can be calculated by

$$
S_{\hat{\dot{\theta}}}=\frac{\hat{u}}{\hat{\dot{\theta}}}, \Delta \phi_{\dot{\theta}}=\frac{180}{\pi} \cdot\left(\phi_{u}-\phi_{\dot{\theta}}\right)
$$

where $\hat{\dot{\theta}}$ and $\phi_{\dot{\theta}}$ are the angular vibration amplitude and the initial phase of the angular vibration, respectively.

The frequency characteristics of this prototype can be drawn according to the experiment results on the angular vibration table. The sensor's amplitude-frequency characteristic and phase-frequency characteristic curve are shown in Figure 8a and b, respectively. In addition, the theoretical amplitude-frequency curve of the model is the blue line in Figure 8a. It can be seen that the amplitude-frequency

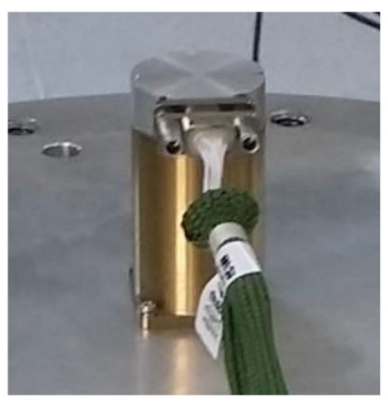

(a)

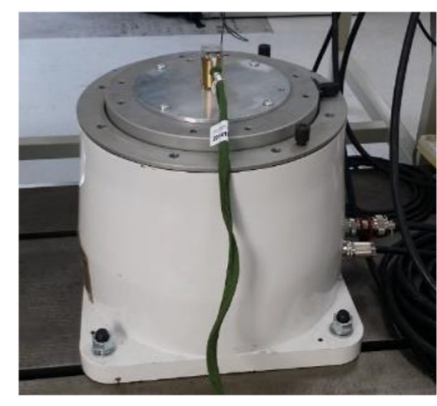

(b)
Fig. 7. (a) Prototype of the MHD angular vibration sensor; (b) Prototype on Angular Vibration Table.

characteristic of the sensor prototype performs as a bandpass filter, and the maximum scale factor is about 40.2 $40.5 \mathrm{~V} / \mathrm{rad} / \mathrm{s}$. Thus, the lower-cut-off frequency $(-3 \mathrm{~dB})$ is about $6.4 \mathrm{~Hz}$, and the scale factor at $1000 \mathrm{~Hz}$, which is still in the pass band.

The experiment shows that the lower-cut-off frequency of this MHD prototype is about $6.4 \mathrm{~Hz}$, which has an $6.7 \%$ error rate. This delay is speculated due to the transformer. From Figure 8a, it can be seen that the scale factor has a significant downward trend at $1000 \mathrm{~Hz}$, but still in the pass band. This is due to the active second-order low-pass filter. So, it can be estimated that the upper-cut-off frequency is not higher than the $1200 \mathrm{~Hz}$, the cut-off frequency of the low-pass filter. The max scale factor of this prototype is $40.5 \mathrm{~V} / \mathrm{rad} / \mathrm{s}$, which is about $1.4 \%$ error from the designed $39.96 \mathrm{~V} / \mathrm{rad} / \mathrm{s}$. This error may be caused by various factors, such as noise, resistance accuracy in the circuit, etc.

\section{Conclusion}

This paper first derives the transfer function of the angular vibration response of the conductive fluid in the static magnetic field. Then, the magnetic field and electric circuit model of the sensor is designed. The model is designed as a high-pass filter with a cut-off frequency of $6 \mathrm{~Hz}$. Based on this model, the sensitivity of the design parameters of magnetic field intensity, conducting fluids' height and width are analyzed to get the optimized parameter for higher precision and width. After that, a prototype, including the measuring circuit, was developed to verify the analysis and optimization. Finally, the experiment is implemented to get the frequency characteristics of $1-1000 \mathrm{~Hz}$. The result shows that the cut-off frequency of the prototype increases from $6 \mathrm{~Hz}$ of the model to $6.4 \mathrm{~Hz}$, and the error is about $6.7 \%$. the max error in scale factor is about $1.4 \%$, which from 39.96 to $40.5 \mathrm{~V} / \mathrm{rad} / \mathrm{s}$. These errors are within the acceptable, and the results can verify the model proposed in this paper. The experiment shows that the prototype meets the design requirement of $6-1000 \mathrm{~Hz}$, and the model is accurate.

However, this MHD angular vibration sensor still needs some improvements. The current MHD prototype cannot measure low-frequency angular vibration signals below $6 \mathrm{~Hz}$. The traditional gyroscope is needed to be used with 


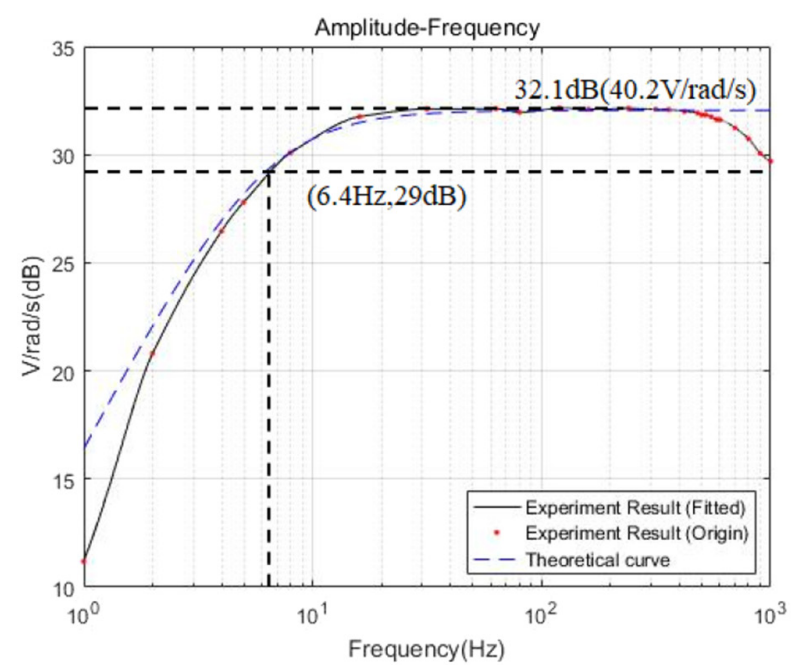

(a)

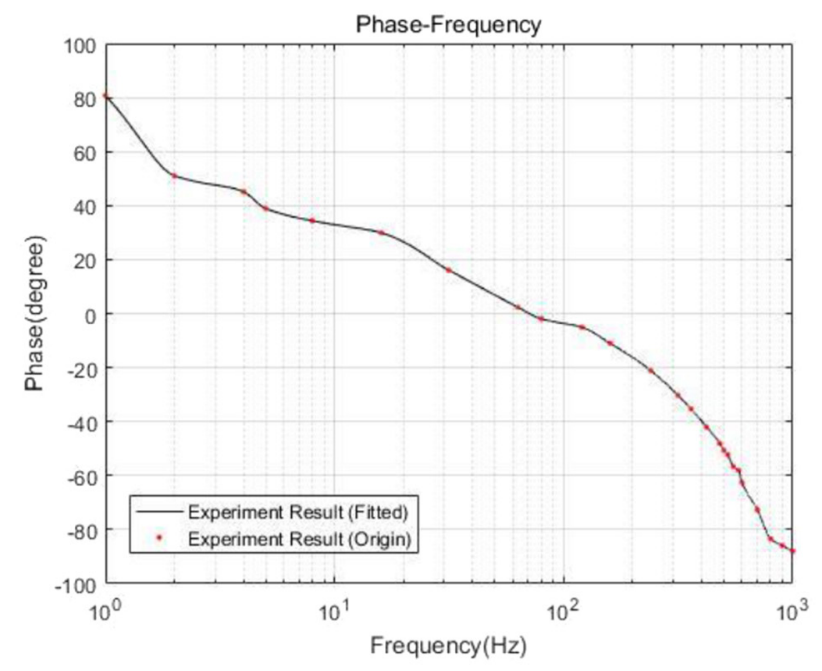

(b)

Fig. 8. Frequency characteristics of MHD sensor prototype in experiment. (a) Amplitude-Frequency; (b) Phase-Frequency.

this sensor prototype to complete angular vibration measurements within $0-1000 \mathrm{~Hz}$. This is mainly because a larger magnetic flux density $B$ is used to improve the signal-to-noise ratio, the output of sensor and reduction circuit amplification, which leads to a higher cut-off frequency in the model. In addition, the transformer in the measuring circuit also increases the cut-off frequency. In the future research, a transformer with better lowfrequency performance should be selected to decrease the cut-off frequency, and the measuring circuit should be modified to improve the detection and amplification of weak signals. As a result, a smaller magnetic flux density $B$ can be used to decrease the cut-off frequency of the sensor. In addition, noise and the accuracy of circuit components also should be concerned to decrease the error.

\section{References}

1. D.R. Laughlin, D. Smith, Development and performance of an angular vibration sensor with $1-1000 \mathrm{~Hz}$ bandwidth and nanoradian level noise, Free-Space Laser Communication and Laser Imaging, Int. Soc. Opt. Photonics 4489, 208-214 (2002)

2. M. Sohail, Modified heat and mass transmission models in the magnetohydrodynamic flow of Sutterby nanofluid in stretching cylinder, Phys. A Stat. Mech. Appl. 549, 124088 (2020)

3. R.V.M.S.S. Kiran Kumar, S. Vijaya Kumar Varma, C.S.K. Raju, S.M. Ibrahim, G. Lorenzini, E. Lorenzini, Retraction Note to: Magnetohydrodynamic 3D slip flow in a suspension of carbon nanotubes over a slendering sheet with heat source/ sink, Contin. Mech. Thermodyn. 29, 1-17 (2019)

4. S. Jimenez-Flores, J.G. Pérez-Luna, J.J. Alvarado-Pulido, A.E. Jiménez-González, Development and simulation of a magnetohydrodynamic solar generator operated with $\mathrm{NaCl}$ electrolyte solution, J. Solar Energy Eng. 143, 1-9 (2020)

5. C.R. Evans, J.F. Hawley, Simulation of magnetohydrodynamic flows - a constrained transport method, Astrophys. J. 332, 659-677 (2007)
6. H. Alfvén, Magnetohydrodynamic waves in the atomic nucleus, Phys. Rev. 107, 632-632 (1957)

7. E. Egorov, V. Agafonov, S. Avdyukhina, S. Borisov, Angular molecular-electronic sensor with negative magnetohydrodynamic feedback, Sensors 18, 245 (2018)

8. M. Anwari, Effect of magnetic field on a Diagonal MHD Accelerator, 2008 IEEE Vehicle Power and Propulsion Conference, IEEE, 2008, pp. 1-5

9. D.R. Laughlin, Magnetohydrodynamic (MHD) actuator sensor. Google Patents (2007)

10. D. Laughlin, H. Sebesta, D. Eckelkamp-Baker, A dual function magnetohydrodynamic(MHD) device for angular motion measurement and control, Adv. Astronaut. Sci. 111, 335-347 (2002)

11. T. Iwata, Precision on-board orbit model for attitude control of the advanced land observing satellite (ALOS), J. Aerospace Eng. 4, 62-74 (2012)

12. A. El-Osery, S. Bruder, D. Laughlin, High-accuracy heading determination, 2013 8th International Conference on System of Systems Engineering, IEEE, 2013, pp. 308-313

13. B. Ando, S. Baglio, A. Beninato, A low-cost inertial sensor based on shaped magnetic fluids, IEEE Trans. Instrum. Meas. 61, 1231-1236 (2012)

14. H.E. Shimin, T. Liang, High-bandwidth measurement based attitude determination, Aerospace Control Appl. 37, 20-25 (2011)

15. H. Huo, M. Ma, Y. Li, J. Qiu, The application of MHD angular rate sensor in aerospace, Vac. Cryogenics 17, 114-120 (2011)

16. H. Huo, M. Ma, Y. Li, J. Qiu, High precision measurement technology of satellite's angle microvibration, Transducer Microsyst. Technol. 3, 4-6 (2011)

17. M. Xu, X. Li, T. Wu, X. Yu, C. Chen, Structure design and experiment study for MHD gyroscope, Chin. J. Sci. Instrum. 36, 394-400 (2015)

18. Y. Wu, X. Li, F. Liu, G. Xia, An on-orbit dynamic calibration method for an MHD micro-angular vibration sensor using a laser interferometer, Sensors 19, 4291 (2019) 
19. Y. Ji, G. Yan, Y. Du, Low-frequency extension design of angular rate sensor based on magnetohydrodynamics, 2020 IEEE 5th Information Technology and Mechatronics Engineering Conference (ITOEC), IEEE, 2020, pp. $182-186$

20. Y. Ji, M. Xu, X. Li, T. Wu, W. Tuo, J. Wu, J. Dong, Error analysis of magnetohydrodynamic angular rate sensor combing with coriolis effect at low frequency, Sensors 18, 1921 (2018)
21. Y. Ji, X. Li, T. Wu, J. Wu, Preliminary study on the magnetohydrodynamic (MHD) angular rate sensor combing coriolis effect at low-frequency, 2017 IEEE 3rd Information Technology and Mechatronics Engineering Conference (ITOEC), IEEE, 2017, pp. 210-214

22. R. Moreau, S. Molokov, H.K. Moffatt, Julius Hartmann and his followers: a review on the properties of the Hartmann layer, in: Magnetohydrodynamics, Springer, 2007, pp. 155-170

Cite this article as: Kundong Wang, Youwei Ma, Jianming Wu, Qinghua Zhu, Yue Gu, Hongli Qi, Design parameters analysis and verification of angular vibration sensor based on magnetohydrodynamics, Int. J. Metrol. Qual. Eng. 12, 2 (2021) 